\title{
Various Techniques Employed in the Removal of Apical Root Tips Following Dental Extraction
}

\author{
Narendar Ramesh ${ }^{1}$, Kavin Thangavel ${ }^{2}$, Ramaraj Jayabalan Aravind ${ }^{3}$, Indra Kumar ${ }^{4}$, Gayathri Priydharshini ${ }^{5}$, Anto Ashmi ${ }^{6}$
}

\begin{abstract}
Tooth extraction is one of the very common procedures performed in a dental setup. Tooth loss might influence the patients' class of life and it is a significant indicator of oral hygiene. However, it is indicated in conditions where a tooth is already fractured or spoiled by dental caries beyond a situation where it could be restored by restorations like endodontic fillings or prosthetic options. Every so often, however, there is excessive harm for the tooth to be repaired. There are many reasons as to why a tooth has to be extracted. Few of them are trauma, periodontal diseases, orthodontic reasons, caries-affected, impactions, supernumerary teeth, and for prosthetic management. Tooth extraction is known to be the deletion of a tooth from its socket in the jaws. There are various techniques employed for the same. Though dentists handle it in a very effective manner, there are few chances of complications and failures to occur. One common failure encountered is the fracture of tooth. It may occur in either the crown portion or the root. Fracture of roots can still be classified into three. Of which this article, deals with the fracture at apical one-third and apical two-thirds of the root. This article deals with various techniques for the removal of the broken apical third or two-third that remains in situ.

Keywords: Endodontic files, Open method, Probe, Root tip removal, Syringe needle.

Annals of SBV (2020): 10.5005/jp-journals-10085-8124
\end{abstract}

\section{INTRODUCTION}

Dental extraction is one among the very few dental managements that has to be considered as the very final decision. Reduced quantity of teeth possible will lead to deprived diet and decline in the class of living. The number of extracted teeth proves to be an indication of the socioeconomic status and oral hygiene condition of the individuals. ${ }^{1}$ Extraction of teeth is generally implemented for more than a few reasons like dental caries, periodontitis, orthodontic reasons, impaction, and other failed dental treatments. ${ }^{2}$ Therefore, the knowledge on it becomes an important component to achieve the best oral hygiene status. Patients progressively keep questioning medical practitioners about the consequences of a surgical method. They have regularly asked if they would be able to resume work post-extraction, when the effect of anesthesia would reduce, when to recommence normal diet, how extensively they would sense pain and at times probable complications post non-surgical tooth extraction. Hence, it is imperative to discover the outcomes of routine tooth extraction. ${ }^{3}$ The most common complications are dry socket (alveolar osteitis), prolonged bleeding, post-extraction swelling, tooth fracture, and maxillary sinus perforation. Alveolar osteitis is a known complication but the exact pathogenesis is not well understood and most concepts are still subject to significant controversy. ${ }^{4}$ Of all these, the tooth fracture could be the mostly encountered one that is strictly under the dentists' control. An array of techniques have been known in the writings for eradication of broken root like crafting a bony window over the root apex ${ }^{5}$ or surgical elimination of bone in the region of the root within the socket and rescuing the root fragment, exclusion with apex elevators, luxators, periotome ${ }^{5}$ syringe needle ${ }^{6}$ or by putting

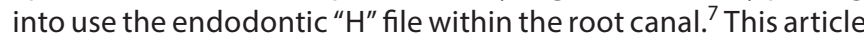
deals about the above-mentioned techniques. $1-4,6$ Department of Oral and Maxillofacial Surgery, Vivekanandha
Dental College for Women, Namakkal, Tamil Nadu, India
${ }^{5}$ Department of Periodontics, Vivekanandha Dental College for
Women, Namakkal, Tamilnadu, India

Corresponding Author: Narendar Ramesh, Department of Oral and Maxillofacial Surgery, Vivekanandha Dental College for Women, Namakkal, Tamil Nadu, India, Phone: +91 9629529581, e-mail: sairamnaren@gmail.com

How to cite this article: Ramesh N, Thangavel K, Aravind RJ, Kumar I, Priydharshini G, Ashmi A. Various Techniques Employed in the Removal of Apical Root Tips Following Dental Extraction. Ann SBV 2020; 9(2):36-38.

Source of support: Nil

Conflict of interest: None

\section{Discussion}

The retained root fragments are more prevalent in edentulous mouths. The prevalence of this is well described with a good number in rats, keeping hold of the apical portion of the root. A majority of the extraction sites healed in a good way, but it was found to be slower than empty extraction sockets owing to the inflammatory processes. It was found that when bone or root fragments were present close to the surface, the occurrence of it in edentulous mouths was finely described with retained roots being the a good number of common findings on radiographs. ${ }^{8}$ The frequency varies among studies, by $15.4-37.3 \%{ }^{8}$ of edentulous patients possessing at least one retained root. The commonness of retained root fragments in partially dentate mouths has been accounted to have a comparatively lower frequency, with incidences of $20 \%,{ }^{9} 13 \%,{ }^{10}$ and $11 \% .{ }^{11}$ Maxillary 
left molars were highly indicative of oroantral problems and root fractures (59.3-60.2\%) when weighed against the maxillary right molars (40.7-39.8\%). ${ }^{10}$ This was attributed the dominance of the operators' hands. When a part of the root fractures occurs at some point during the extraction, it can be left behind and it is common in day-to-day practice. So when such a situation arises, there is a different approach from usual curing. It exhibits changes in the length, ${ }^{12}$ pulpal status, ${ }^{13}$ and position ${ }^{14}$ and sufficient closure of the socket boundaries ${ }^{15}$ as well.

Glickman et al., ${ }^{16}$ and Smith ${ }^{17}$ have performed animal trials by retaining a fragment of tooth root in the socket in animals. They had used albino rats for the same. The observation was that the sites healed well; however, in a slower rate when compared to the empty sockets and this was owing to the inflammatory processes. Moreover, it was noted that while the fragments lied in near proximity to the surface, the epithelium was broken and they extruded with no trouble. Fragments that were found to be profound inside the socket were not externalized, and this was because of the inability of the epithelium to not expand beneath the root fragment completely. The deeper fragments and the surrounding inflammatory cells that lie in the socket, become open to the elements of the oral microbial flora, and eventually build up periapical abscesses. ${ }^{16,17}$ So, after performing the risk benefit analysis, the decisions are to be made for if the root tip should be retained or not. Because, there are undoubtedly circumstances while leaving the root is the most prudent and sound clinical decision one can make. ${ }^{18}$ Some of the risks associated with the elimination of apical third or two-third of the root are pushing of it into the antrum or lingual pouch, damage or injury to the nearby tooth or teeth and structures that are vital, alveolar osteitis, and oroantral fistula. ${ }^{19}$ Similarly, some of the benefits associated are that the prevalence of vital roots have publicized to preserve alveolar bone for prosthodontic intentions. ${ }^{20}$ Supremely, conventional root filling materials will not be present to cause an seditious response. Implants show successful integration in the region of vital root fragments with cementum apposition occurring in the interface between the implants and the vital root in situ. ${ }^{21}$ Nevertheless, it is every so often complicated to make certain the situations in places where a fractured root segment is vital or not. Therefore, the judgment to get rid of retained root that have broken intraoperatively at any point in the duration of tooth extraction procedures requires to be performed on a case-by-case source. The pragmatic approach of "non nocere"-_ does no harm" is to be followed. So for cases where the root fragment has to be removed, there are various techniques to achieve the same. Some of them are, creating a bony window over the apex ${ }^{5}$ or surgical exclusion of bone in the region of the root inside the socket and eradicating the root fragment, amputation with apex elevators, luxators, periotome ${ }^{5}$ syringe needle ${ }^{6}$ or with the use of endodontic " $\mathrm{H}$ " file within the root canal. ${ }^{7}$ A novel technique was then developed which is atraumatic for the removal of broken root tip of maxillary premolars. This technique is based on the thinness of buccal cortical plate of maxillary premolar area. ${ }^{22}$

\section{Open Method}

An incision is made and reflection of the flap is performed in the area where the tooth has to be removed. Removal of the buccal cortical bone along the length of the root is made preferably using a round bur. Luxation should be performed now using a straight elevator. Smoothening of the bone margins is performed with a bone file. Suturing is now performed.

\section{Using LA Needle}

If the root is luxated prior to the fracture during extraction, and is found to be tapered apically, a normal 25-gauge needle that is used for routine local anesthesia administration can be used. Typically, the needle will be retentive enough in the canal to eliminate the retained root without causing further damage and compromise to the adjacent vital tissues. ${ }^{6}$

\section{Using Hedstrom Files}

An attempt is made to remove the part of the root by suction. However, when it becomes not possible, identification of the root canal is initially made. Screwing of the No. $35 \mathrm{H}$-file should be made until it is tight into the canal (in such a way that the file does not break off). The better we place the file in correct position, the better the situation is in control. Now, tugging of the file should be made. In most cases, it is expected to be retrieved. However, if it does not come out, other methods of removal can be performed. ${ }^{7}$

\section{Using K Files}

After insertion of a suitable number file in to the canals, first 3/4th of a clockwise rotation is to be made. It should be checked for tug back. At this point, a $1.5 \mathrm{~mm}$ insertion of file into the root piece is expected. Force must be delivered toward apical direction with one-fourth of a rotation that is performed clockwise. This in turn would aid to produce rubber band-elastic effect by means of apical periodontal fibers. This repels the tip of the root in opposite way. The clockwise rotation assists in tearing of the lateral fibers attached to cortical plates. All these movements result in the removal of the root piece. ${ }^{23}$

\section{Using a Probe}

This procedure is engaged for roots that are broken down with bevel facing toward the buccal cortex. An envelope flap should be made on the buccal side corresponding to the tooth. A sharp probe should be inserted into the socket. Now the probe's tip has to be heading toward the buccal cortex all along the course of the bevel of the broken root. The probe must slightly be moved forward with tender strength till it enters and pierces the complete thickness of the buccal cortex, thereby making an aperture and its tip becomes noticeable buccally. The probe can now be withdrawn from the socket. It has to be then inserted into the aperture outwardly to capture the fractured root and then downward force is put forth to shove the tip of the root occlusally, thereby facilitating its elimination. 3-0 braided black silk sutures are to be placed. ${ }^{24}$

A brief summary about a powered periotome that has been developed to support in the atraumatic extraction of teeth and another new-fangled mechanism, the piezosurgery, ever more that was employed for outpatient oral surgery procedures are discussed by Dym and Weiss in his article.

\section{Conclusion}

If only the apical one-third (3-4 $\mathrm{mm}$ ) of the root breaks through a closed extraction, a methodical practice should be employed to eliminate the root tip. Preliminary efforts are to be made to take out the root fragment from the place by a closed technique, but the surgeon could begin a surgical technique if the closed method 
is not right away victorious. The pragmatic approach of "non nocere"-_does no harm" has to be kept in mind by all the dentists.

\section{References}

1. Chrysanthakopoulos NA. Reasons for extraction of permanent teeth in Greece: a five-year follow-up study. Int Dent J 2011;61(1):19-24. DOI: 10.1111/j.1875-595X.2011.00004.x.

2. Jafarian M, Etebarian A. Reasons for extraction of permanent teeth in general dental practices in Tehran, Iran. Med Princ Pract 2013;22(3):239-244. DOI: 10.1159/000345979.

3. Adeyemo WL, Taiwo OA, Oderinu OH, Adeyemi MF, Ladeinde AL, Mobolanle $\mathrm{O}$. Ogunlewe oral health-related quality of life following non-surgical (routine) tooth extraction: a pilot study. Contemp Clin Dent 2012;3(4):427-432. DOI: 10.4103/0976-237X.107433.

4. Kolokythas A, Olech E, Miloro M. Alveolar osteitis: a comprehensive review of concepts and controversies. Int J Dent 2010;2010:249073. DOI: $10.1155 / 2010 / 249073$.

5. Harry D, Adam W. Exodontia: tips and techniques for better outcomes. Dent Clin North Am 2012;56(1):245-266. DOI: 10.1016/j. cden.2011.07.002.

6. Orr 2nd DL, Haworth G. Root tip recovery via local anesthetic needle. Oral Surg Oral Med Oral Pathol 1985;60(2):158. DOI: 10.1016/00304220(85)90284-1.

7. Stoner Kenneth E. Using a Hedstrom endodontic file to retrieve a root tip. J Am Dent Assoc 2002;133(4):473. DOI: 10.14219/jada. archive.2002.0206.

8. Sumer AP, Sumer M, Guler AU, Bicer I. Panoramic radiographic examination of edentulous mouths. Quintessence Int 2007;38(7):e399e403.

9. Dachi SF, Howell FV. A survey of 3,874 routine fullmouth radiographs. I. A study of retained roots. Oral Surg Oral Med Oral Pathol 1961;14:916924. DOI: $10.1016 / 0030-4220(61) 90003-2$.

10. Kharat DU, Saint T. Embedded root fragments in completely and partially edentulous jaws. Saudi Dent J 1991;3:8-12.

11. Bayanzadeh S, Shokri S. Radiographic evaluations of patients seeking removable prostheses treatment. J Dent Med 2004;16:78-81.
12. Simpson HE. The healing of extraction wounds. Br Dent $J$ 1969;126(12):550-557.

13. Whitaker DD, Shankle RJ. A study of the histologic reaction of submerged root segments. Oral Surg Oral Med Oral Pathol 1974;37(6):919-935. DOI: 10.1016/0030-4220(74)90445-9.

14. Herd JR. The retained tooth root. Aust Dent J 1973;18(3):125-131. DOI: 10.1111/j.1834-7819.1973.tb03448.x.

15. Pietrokovski J. Extraction wound healing after tooth fracture in rats. J Dent Res 1967;46(1):232-240. DOI: 10.1177/00220345670460011601.

16. Glickman I, Pruzansky S, Ostrach M. The healing of extraction wounds in the presence of retained root remnants and bone fragments: an experimental study. Am J Orthodontics Oral Surg 1947;33(4):263-283. DOI: 10.1016/0096-6347(47)90063-X.

17. Smith RL. The role of epithelium in the healing of experimental extraction wounds. J Dent Res 1958;37(2):187-194. DOI: 10.1177/00220345580370020201.

18. Leonard M. The root tip: to pursue or not to pursue. Dent Today 2001;20(10):108.

19. Nayyar J, Clarke M, O'Sulliva M, Stassen LFA. Fractured root tips during dental extractions and retained root fragments. A clinical dilemma? Br Dent J 2015;218(5):285-290. DOI: 10.1038/sj.bdj.2015.147.

20. Casey DM, Lauciello FR. A review of the submerged-root concept. J Prosthet Dent 1980;43(2):128-132. DOI: 10.1016/0022-3913(80)901742.

21. Hurzeler MB, Zuhr O, Schupbach P, Rebele SF, Emmanouilidis $N$, Fickl S. The socket-shield technique: a proof-of-principle report. J Clin Periodontol 2010;37(9):855-862. DOI: 10.1111/j.1600051X.2010.01595.x.

22. Histology Antonio Nanci ten Cate's oral. Development, Structure, and Function. Amsterdam: ElsevierHealth Sciences; 2008.

23. Abhishek T, Vinay U, Chandurkar K, Rohit K, Anirudh R. Atraumatic technique for removal of broken root tips of maxillary posterior teeth with endodontic K file-A technical consideration. J Dent Oral Disord Ther 2018;6(2):1-4.

24. Singh C, Sharma D, Newaskar V, Agrawal D. A simple technique for removal of broken root tip of maxillary premolars: a technical note. J Maxillofac Oral Surg 2015;14(3):866-867. DOI: 10.1007/s12663-0140696-7. 